Trauma Berufskrankh 2012 • 14[Suppl 3]:347-351 DOI 10.1007/s10039-011-1824-y

Online publiziert: 22. März 2012

๑) Springer-Verlag 2012

A. Nusche $\cdot$ H.-E. Schaller

Klinik für Plastische, Hand-, Rekonstruktive und Verbrennungschirurgie, Eberhard Karls Universität Tübingen, BG-Unfallklinik Tübingen

\title{
Rehabilitation nach schwerer Handverletzung
}

letzung mit Beugesehnen- und Nervendurchtrennungen dargestellt.

\section{Fallbeispiel}

Der im Folgenden dargestellte rehabilitative Ablauf bezieht sich auf eine 24-jährige Patientin mit einer tiefen queren Schnittverletzung in der proximalen Hohlhand mit Durchtrennung aller 9 Beugesehnen, des $\mathrm{N}$. medianus und des $\mathrm{N}$. ulnaris (• Abb. 1).

\section{Nachbehandlung nach Kleinert}

\section{1. bis 4. Woche}

Nach der operativen Versorgung mit Naht aller durchtrennten Strukturen wird am 3. postoperativen Tag (ohne Nervenverletzungen am 1. postoperativen Tag) in der Abteilung für Ergotherapie die KleinertSchiene (- Abb. 2) angefertigt, welche eine frühfunktionelle Beübung erlaubt. Diese Therapie nach Kleinert wurde erst- mals 1955 beschrieben [1, 2]. Durch sie wird eine Verklebung der genähten Beugesehnen untereinander und mit dem umgebenden Gewebe verhindert.

Die Kleinert-Schiene wird aus thermoplastischem Material (Polyform) in $30^{\circ}$ Beugung des Handgelenks und $60^{\circ} \mathrm{Beu}-$ gung der Langfingergrundgelenke streckseitig angefertigt, um die genähten Beugesehnen zu entlasten. Die Schiene endet streckseitig in Höhe der Mittelgelenke.

Um eine frühfunktionelle Beübung zu ermöglichen, werden die Finger, an denen Beugesehnen genäht wurden, mit an den Fingernägeln befestigten Zügeln versehen. Sind eine oder mehrere tiefe Beugesehnen der ulnaren 3 Langfinger betroffen, werden wegen des gemeinsamen Muskelbauchs alle 3 ulnaren Langfinger mit Zügeln versehen. Am proximalen Ende dieser Zügel befinden sich Federn, die die Finger nach ihrer aktiven Streckung wieder in die Beugung ziehen und damit die genähten Beugesehnen entlasten. Die Fe-

$\mathrm{Zu}$ den wesentlichen Bestandteilen der medizinischen Rehabilitation gehören

- Physiotherapie

- Ergotherapie

- Schmerztherapie

- Psychologische Behandlung

- Entlassmanagement

Um ein möglichst gutes funktionelles Ergebnis nach schweren Handverletzungen mit Sehnen- und Nervendurchtrennungen zu erreichen, müssen die oben genannten Rehabilitationsmaßnahmen intensiv und interdisziplinär über einen langen Zeitraum eingesetzt werden. Anhand des nachfolgenden Beispiels wird der Rehabilitationsablauf nach einer Handver-

Abb. 1 - Tiefe Schnittverletzung quer durch die Hohlhand mit Durchtrennung aller Beugesehnen, des N. medianus (auf Kompresse) und des N. ul-

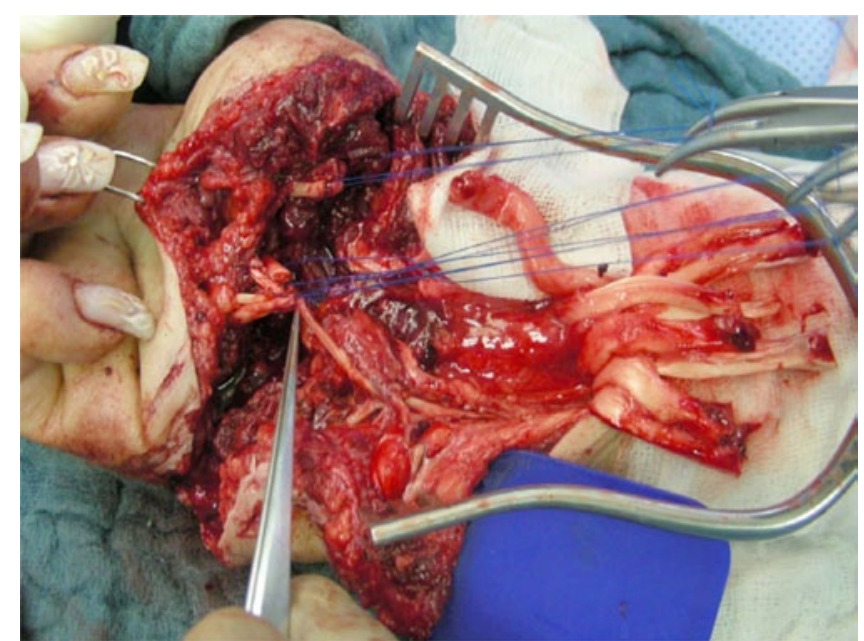

naris 


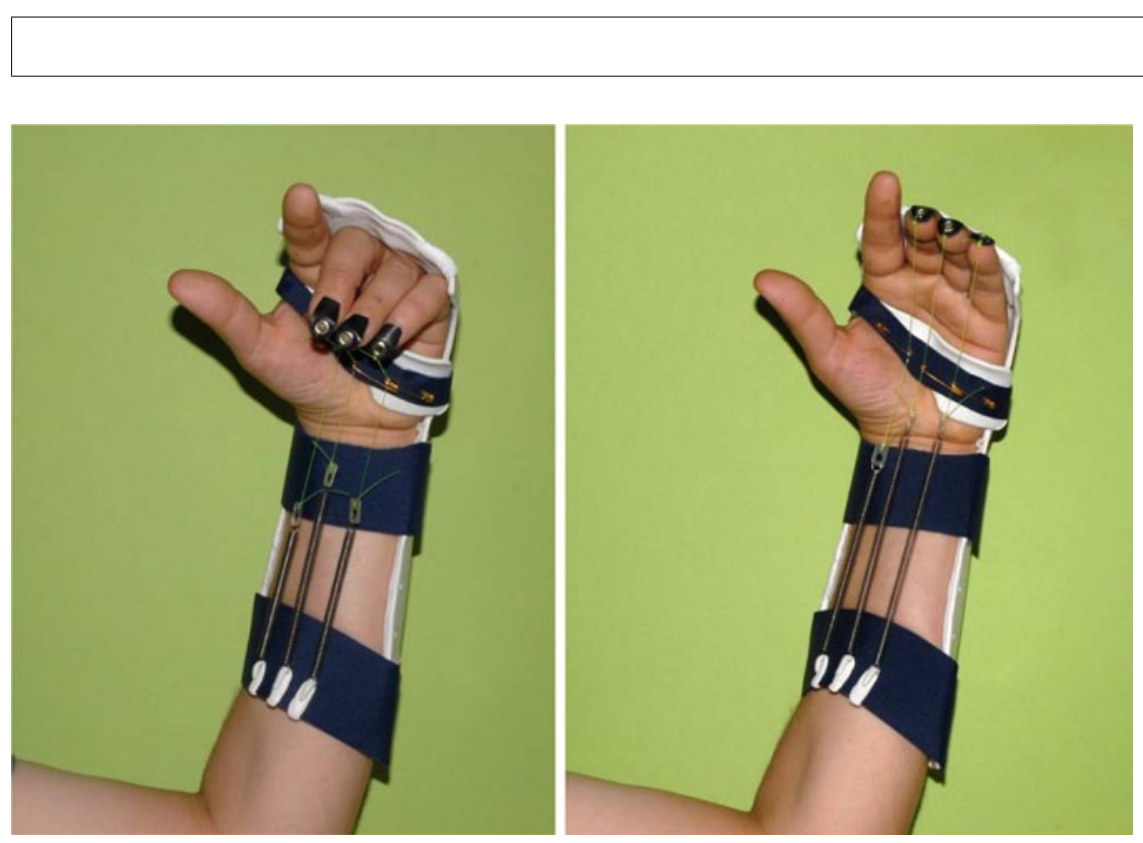

Abb. $2 \Delta$ Beispiel einer Kleinert-Schiene für die ulnaren 3 Langfinger

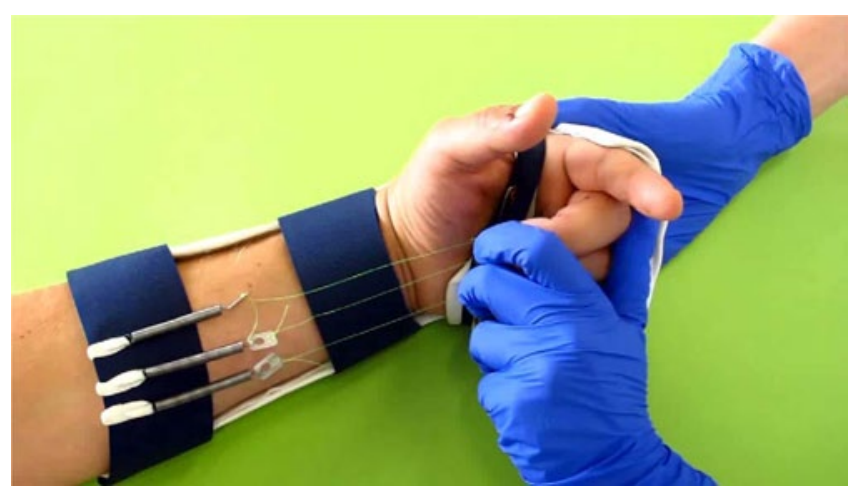

Abb. $3<$ Physiotherapie mit angelegter Kleinert-Schiene: passive Verstärkung der Beugung durch den Therapeuten
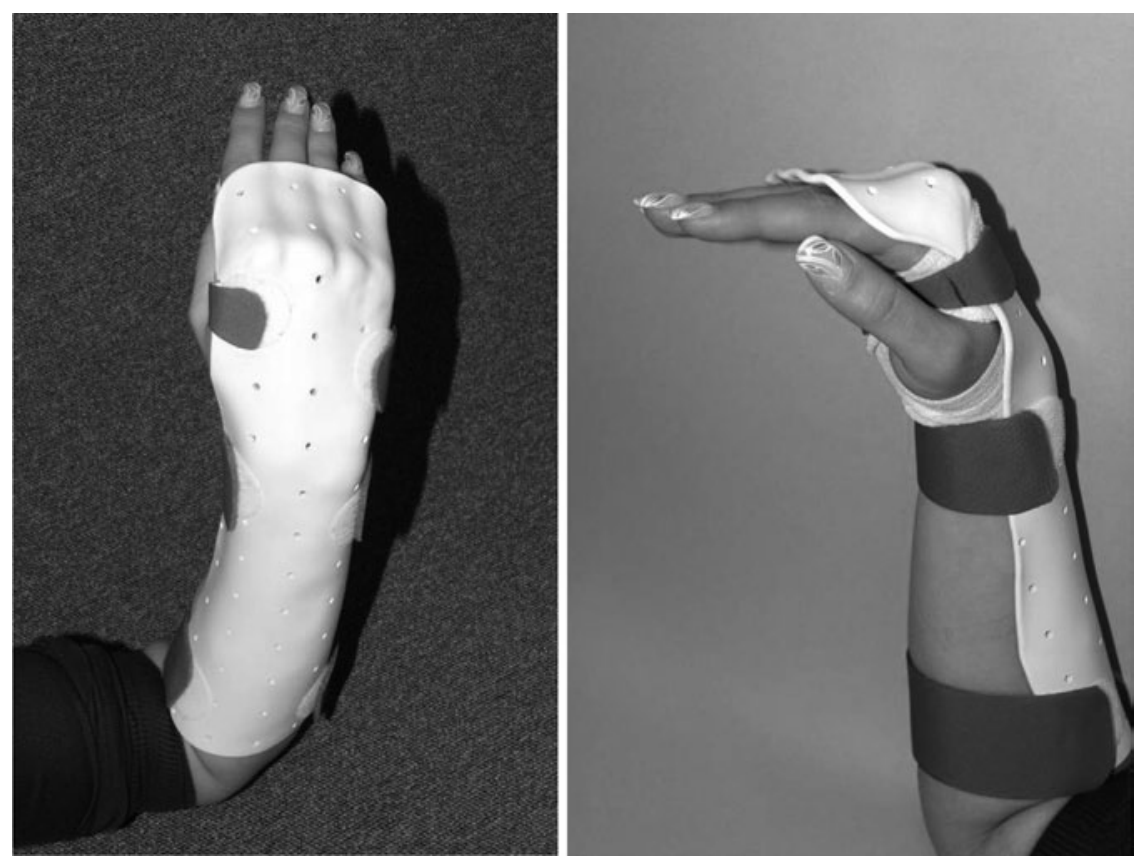

Abb. 4 \ Kleinert-Schiene ohne Zügel (5. und 6. Woche)

\section{Zusammenfassung $\cdot$ Abstract}

Trauma Berufskrankh 2012 · 14[Suppl 3]:347-351 DOI 10.1007/s10039-011-1824-y

(c) Springer-Verlag 2012

\section{A. Nusche $\cdot$ H.-E. Schaller \\ Rehabilitation nach schwerer Handverletzung}

\section{Zusammenfassung}

Im vorliegenden Beitrag wird der rehabilitative Ablauf nach einer tiefen queren Schnittverletzung in der proximalen Hohlhand mit Durchtrennung aller 9 Beugesehnen, des N. medianus und des N. ulnaris am Beispiel einer 24-jährigen Patientin dargestellt. Hierbei wird insbesondere auf die funktionelle Nachbehandlung nach Kleinert, Abhärtungsmaßnahmen, die Überprüfung der Reinnervierung und das Sensibilitätstraining eingegangen.

\section{Schlüsselwörter}

Handverletzungen · Rehabilitation .

Sehnenverletzungen .

Nervendurchtrennungen $\cdot$ Kleinert-Schiene

\section{Rehabilitation after severe trauma of the hand}

\section{Abstract}

This article deals with rehabilitation after a deep transverse gash in the proximal palm with additional transection of all nine flexor tendons and of the median and ulnar nerves using the example of a 24-year-old female patient. The focus is set on the functional after-treatment according to Kleinert, on hardening techniques, on the examination of reinnervation and on sensitivity training.

\section{Keywords}

Hand injuries · Rehabilitation .

Tendon injuries - Nerve transection .

Kleinert splint

dern sollen eine Zugwirkung von 25-50 g aufweisen. Die Zügel werden mit kleinen Lederflicken, die mit Sekundenkleber auf den Fingernagel aufgeklebt werden, befestigt. Diese Lederflicken enthalten Metallösen, durch die der Zügel geknotet wird. Wichtig ist eine Umlenkung des Zügelzugs in die Hohlhand durch ein dort befindliches Klettband, damit es zu einer vollständigen Beugung der Langfingerendgelenke kommen kann. Die Umlenkung erfolgt mit Hilfe von Sicherheitsnadeln, die an dem Klettband befestigt werden. 

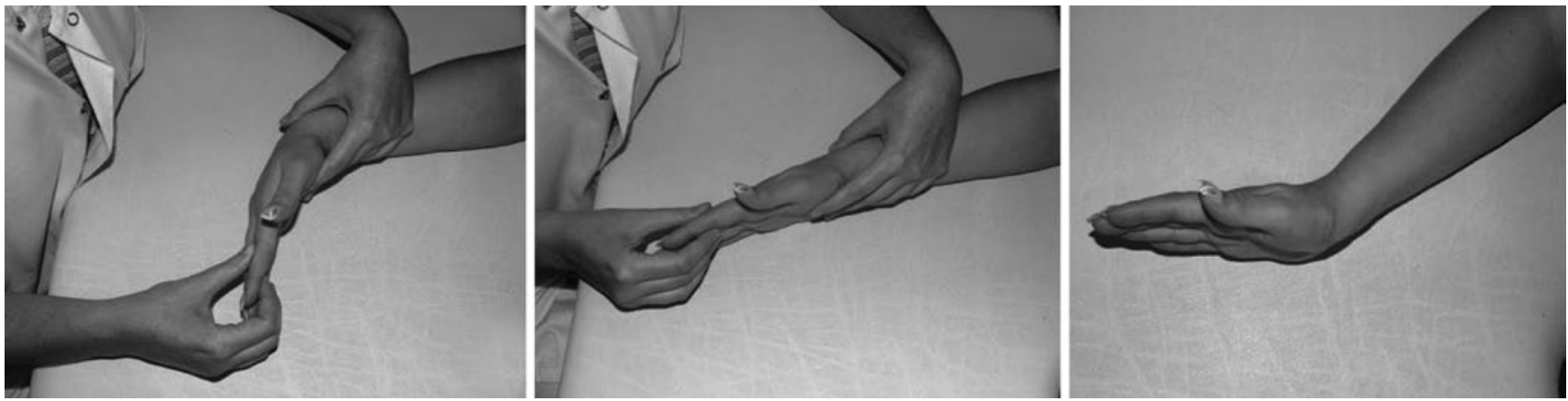

Abb. $5 \Delta$ 7. bis 9. Woche: Physiotherapie bei zunehmender Streckstellung im Handgelenk
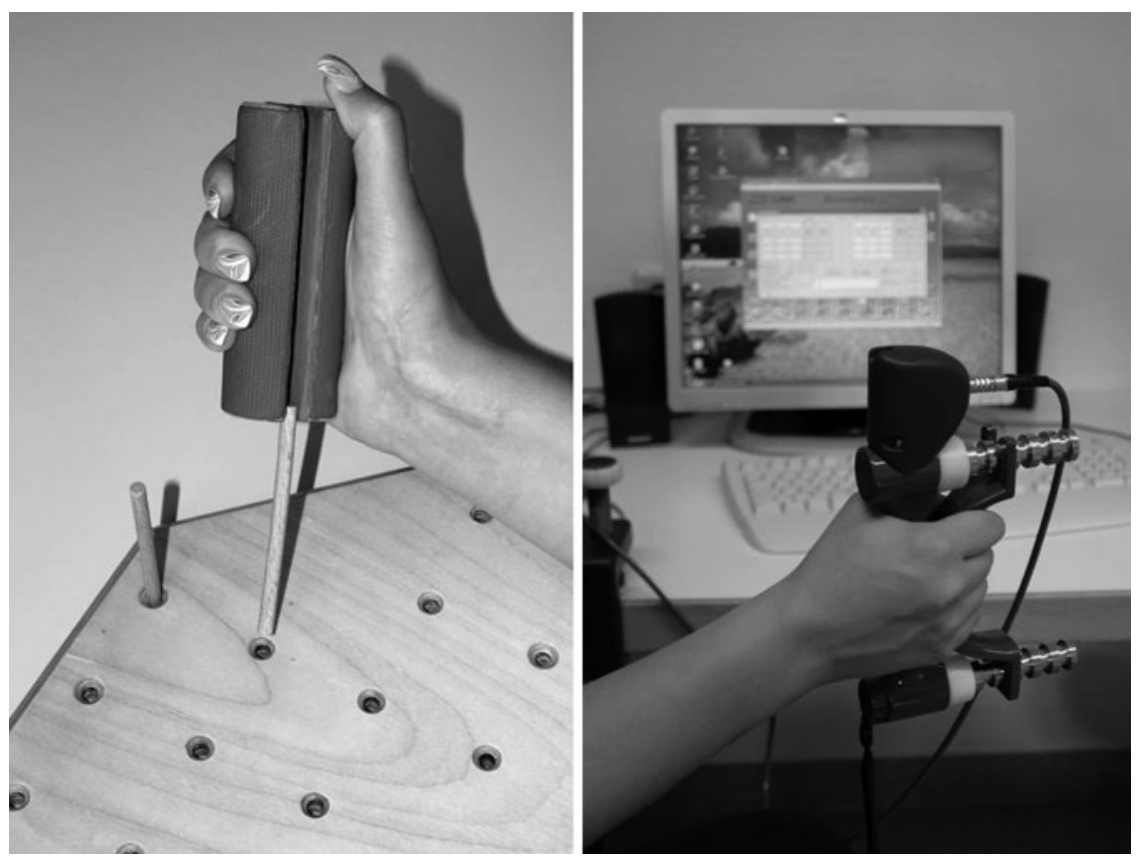

Abb. $6 \Delta$ Zunehmender Kraftaufbau in der 10. bis 12. Woche bis zur Vollbelastung

Nach Fertigstellung der Schiene wird sofort mit der Physiotherapie begonnen. Dabei müssen die Mittel- und Endgelenke durch die intakten Strecksehnen voll gestreckt werden, um Bewegungseinschränkungen zu verhindern. Die Beugung der Langfingergelenke erfolgt sowohl kombiniert als auch einzeln durch Widerlagerung proximal der Gelenke. Sie wird passiv durch die unverletzte Hand des Patienten verstärkt, um eine vollständige Beugung der Fingergelenke zu erreichen (• Abb. 3).

Nach Anleitung in der Physiotherapie werden die einzelnen Übungen vom Patienten 10-mal stündlich durchgeführt. Hierzu sollten der bzw. die betroffenen Langfinger einmal aktiv gestreckt und dann passiv über den/die Zügel ge- beugt werden. Anschließend werden alle Langfinger der betroffenen Hand mit Hilfe der Gegenhand passiv zum kompletten Faustschluss gebracht, d. h. Grund-, Mittel- und Endgelenke werden komplett gebeugt. Abschließend werden nach Abhängen des Zügels bzw. der Zügel die Mittelund Endgelenke des/der betroffenen Finger aktiv auf maximal $20^{\circ}$ gebeugt. Ein aktives Beugen über $20^{\circ}$ hinaus ist hierbei unbedingt zu vermeiden. Bei adäquatem Sitz der angespassten Kleinert-Schiene weisen die Kuppen der angezügelten Finger bei Beugung auf die Umlenkungsstelle der Zügel auf Höhe der Langfingergrundgelenke. Dieses Behandlungsregime führen wir über 4 Wochen durch.
5. und 6. Woche

$\mathrm{Zu}$ Beginn der 5. Woche werden die Zügel entfernt (• Abb.4), und die Beübung der Finger wird ohne Zügel fortgeführt, wobei die Beugesehnen noch bis zum Ende der 6. Woche durch die Kleinert-Schiene entlastet werden.

\section{7. bis 9. Woche}

$\mathrm{Zu}$ Beginn der 7. Woche wird die KleinertSchiene entfernt und die Physiotherapie zunächst noch in entlastender Beugestellung des Handgelenks durchgeführt. Zu Beginn der 8. Woche ist die Nullstellung, ab der 9. Woche auch eine Streckstellung im Handgelenk erlaubt ( Abb. 5).

\section{0. bis 12 . Woche}

$\mathrm{Ab}$ der 10. Woche darf auch gegen Widerstand gebeugt werden. Während zunächst noch weiche Materialien, z. B. Softball oder Therapieknetmasse, zur Anwendung kommen, wird ab der 11 . Woche mit unterschiedlich starken Faustschlussklammern geübt. $\mathrm{Ab}$ der 12 . Woche ist die Vollbelastung erlaubt (• Abb. 6).

\section{Weitere Maßnahmen}

Begleitend werden Abhärtungsmaßnahmen, Narbenmassage und Sensibilitätstrainings durchgeführt.

\section{Abhärtungsmaßnahmen}

Wunden und Narben sind insbesondere an der Hand zu Beginn übermäßig berührungsempfindlich, was mit der Gefahr einer Vermeidungsstrategie und nachfolgender Bewegungseinschränkung einhergeht. Deshalb muss der initial vermehrten Berührungsempfindlichkeit von Anfang an entgegengewirkt werden. Wichtigste Voraussetzung hierbei ist, so früh 


\section{Die schwere kombinierte Handverletzung}
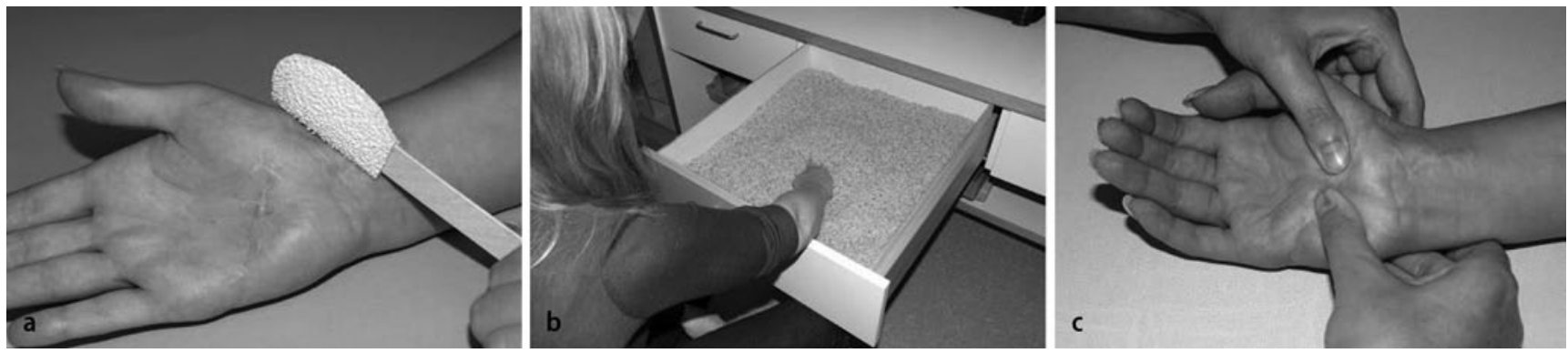

Abb. $7 \Delta$ Abhärtungsmaßnahmen (a,b) und Narbenmassage (c)
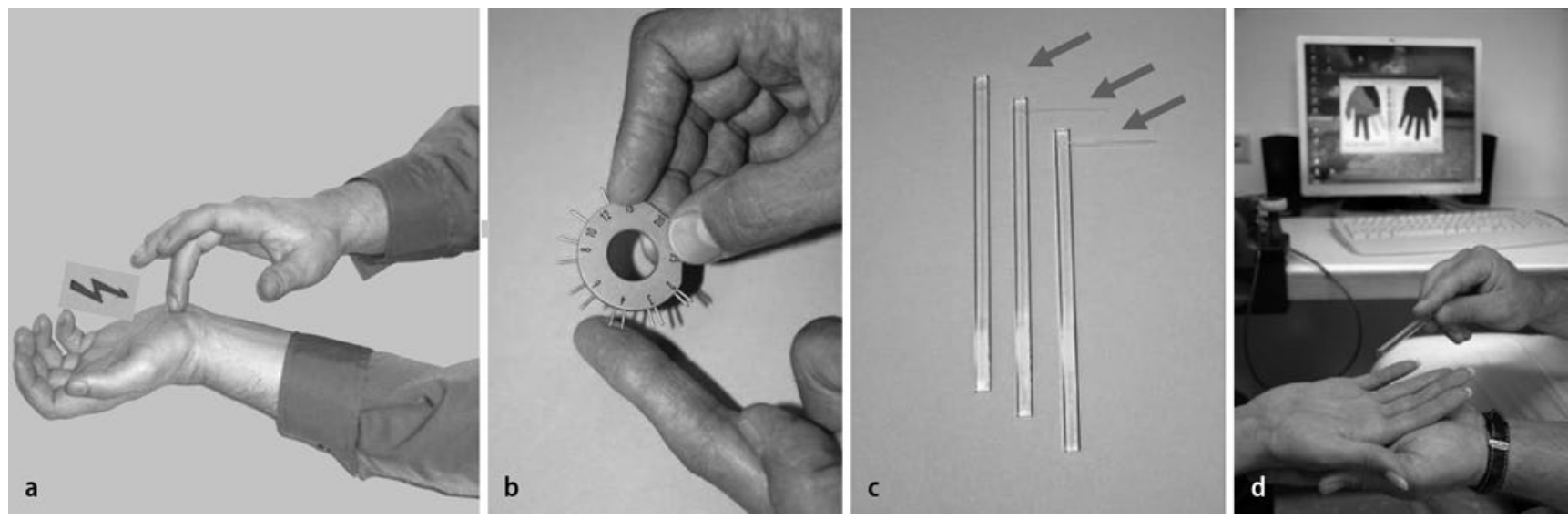

Abb. 8 \ Hoffmann-Tinel-Zeichen (a), 2-Punkte-Diskriminator (b), Semmes-Weinstein-Filamente (c,d)

wie möglich auf die Wunde bzw. die Narbe bedeckende Verbände zu verzichten. Dann kann bereits wenige Tage nach der Operation die Wunde bzw. Narbe z. B. mit einem Abhärtestab mit zunehmender Intensität berührt werden. Dem gleichen Ziel dienen Handbäder mit Linsen, Perlen, Nudeln (• Abb. 7a,b) oder Ähnlichem. Der Fantasie sind diesbezüglich keine Grenzen gesetzt.

Insbesondere nach begleitenden Nervenverletzungen ist die Hand wegen der gestörten Gefäßinnervation vermehrt kälteempfindlich. Wechselbäder und vorsichtige Eisanwendungen trainieren die gestörte Vasomotorik.

\section{Massage}

Die begleitende Narbenmassage (- Abb.7c) soll neben der Abhärtung auch die Narbe weicher machen und Verklebungen in der Tiefe lösen.

\section{Reinnervierung und Sensibilitätstraining}

Die fortschreitende Reinnervierung wird mit dem Hoffmann-Tinel-Zeichen (- Abb. 8a) überwacht, indem der ko- aptierte Nerv von distal nach proximal auf die Nervennaht zu beklopft wird. Der Ort der maximalen Reizantwort wird genau dokumentiert. Im Idealfall kann von einer Reinnervierungsgeschwindigkeit von $1 \mathrm{~mm}$ pro Tag ausgegangen werden. Stagniert das Hoffmann-Tinel-Zeichen, muss der Nerv revidiert und ggf. neurolysiert oder transplantiert werden [3].

Ist die Reinnervierung distal am Erfolgsorgan angekommen, kann die wiederhergestellte Sensibilität mit dem 2-Punkte-Diskriminator ( $\bullet$ Abb. 8b) oder besser den Semmes-Weinstein-Filamenten ( Abb. 8c,d) beurteilt werden.

Bei der 2-Punkte-Diskrimination soll der Patient mit geschlossenen Augen angeben, ob er 2 gleichzeitig in unterschiedlichem Abstand aufgesetzte Berührungsreize unterscheiden kann. Nachteil dieser Methode ist der vom Untersucher unterschiedlich ausgeübte Druck.

Demgegenüber erlaubt die Sensibilitätsprüfung mit den Semmes-Weinstein-Filamenten eine wesentlich genauere und objektivere Erfassung der Berührungsperzeption. Das Semmes-Weinstein-Ästhesiometer enthält einen Satz von 20 kalibrierten Untersuchungsstäben (- Abb. 8c). Jeder Stab ist mit einem Nylonfaden unterschiedlicher Dicke (Nr. 1,65-6,65) versehen. Die einzelnen Filamente werden jeweils senkrecht auf das zu untersuchende Hautareal aufgesetzt, bis sie sich verbiegen. In Abhängigkeit vom Filamentdurchmesser ist eine genau definierte Kraft für die Verbiegung des Filaments erforderlich. Somit kann der ausgeübte Druck präzise quantifiziert werden

\section{(- Abb. 8d, [4]).}

Das endgültige Reinnervierungsergebnis kann häufig erst nach Ablauf von 2 Jahren beurteilt werden.

Das Sensibilitätstraining beginnt nach der operativen Wiederherstellung des Nervs, sobald eine streichende Berührung oder konstanter Druck an den Fingern wahrgenommen wird. In der frühen Phase sollen spezifische Empfindungen, z. B. die Unterscheidung zwischen fortgeleiteter (streichender) und konstanter Berührung (Druck), wiedererlernt werden. Hierbei wird die Hand mit dem Ende des Bleistifts, an dem sich der Radiergummi befindet, von der Hohlhand bis zur Fingerkuppe erst streichend, dann punkt- 
förmig drückend berührt. Während der Patient zunächst diese Berührung beobachtet, soll er danach die Augen schließen, sich auf die Berührung konzentrieren und in Worten laut wiederholen, was er tatsächlich spürt.

Die spätere Phase des Sensibilitätstrainings beginnt etwa 6 bis 8 Monate nach der Operation, wenn die streichende und drückende Berührung ganz sicher bis zur Fingerspitze wahrgenommen, lokalisiert und unterschieden werden kann. In dieser Phase sollen selbst kleinste Gegenstände nach ihrer Materialbeschaffenheit, Größe und Gestalt erkannt und unterschieden werden können. Hierbei kommen unterschiedlich raue bis $\mathrm{zu}$ vollkommen glatte Oberflächen, verschiedene Formen, Größen und Materialien zur Anwendung (z. B. Schmirgelpapier unterschiedlicher Körnung, diverse Gegenstände aus Glas, Holz, Plastik, verschiedene Metalle usw.).

\section{Fazit für die Praxis}

\section{Ein gutes Rehabilitationsergebnis nach schwerer Handverletzung kann nur er- zielt werden, wenn die Maßnahmen der medizinischen Rehabilitation, wie Phy- siotherapie, Ergotherapie, Schmerzthera- pie, und die psychologische Behandlung von Anfang an mit allen geeigneten Mit- teln und in interdisziplinärer Zusammen- arbeit durchgeführt werden.}

\section{Korrespondenzadresse

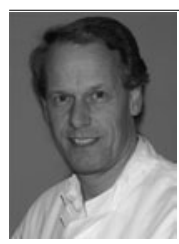 \\ A. Nusche \\ Klinik für Plastische, Hand-, \\ Rekonstruktive und \\ Verbrennungschirurgie, \\ Eberhard Karls Universität \\ Tübingen, BG-Unfallklinik \\ Tübingen, \\ Schnarrenbergstraße 95, \\ 72076 Tübingen \\ anusche@bgu-tuebingen.de}

Interessenkonflikt. Der korrespondierende Autor gibt an, dass kein Interessenkonflikt besteht.

The supplement containing this article is not sponsored by industry.

\section{Literatur}

1. Kleinert HE, Weiland AJ (1979) Primary repair of flexor tendon injuries in zone II. In: Verdan C (Hrsg) Tendon surgery of the hand. Chruchill Livingstone, Edinburgh London New York

2. Kleinert HE, Kutz JE, Ashbell TS, Martinez E (1967) Primary repair of lacerated flexor tendons in „noman's land". J Bone Joint Surg Am 49:577

3. Schaller HE (2006) Nervenrekonstruktion und Nervenersatzoperation. Trauma Berufskrankh [Suppl 1] $8: 28-30$

4. Waldner-Nilsson B, Diday-Nolle AP, Breier S, Slatosch Wintsch DU, Reiter Eigenheer A (2009) Handrehabilitation für Ergo- und Physiotherapeuten, $\mathrm{Bd}$ 1: Grundlagen, Erkrankungen. Springer, Berlin Heidelberg New York, S 42-43 